\title{
Improving patient outcomes in heart failure: evidence and barriers
}

\author{
John G F Cleland
}

Heart failure affects up to $2 \%$ of the adult population. ${ }^{1}$ Interestingly, this rate is remarkably consistent in different populations throughout the world. It does not matter how affluent a society is, the rate of heart failure does not seem to change. In fact, it may be that more affluent societies have more heart failure. It is a disease of the elderly and, in an affluent society, where average life expectancy is increasing, levels of heart failure will also increase. In societies that are not affluent and cardiovascular risk factors are not controlled, the prevalence of heart failure will also be high.

Approximately $50 \%$ of patients with the most severe heart failure (New York Heart Association (NYHA) class IV) die within one year of diagnosis. ${ }^{2}$ In fact, patients diagnosed with heart failure have a three times higher chance of dying within three years than patients diagnosed with breast cancer. If heart failure was a cancer, it would be treated much more effectively. There is a huge fear culture surrounding cancer which demands attention and sucks in resources, but few cardiologists discuss, and few patients with heart failure know, that the prognosis is worse than for most cancers and thus heart failure is not considered a deadly disease in the same way.

\section{Barriers to management}

SIZE OF THE PROBLEM

One of the barriers to the management of heart failure is the sheer size of the problem. In addition to those patients with left ventricular systolic dysfunction and signs of heart failure, there are an equal number of patients who have badly damaged hearts, with major left ventricular systolic dysfunction, but without symptoms. It is also thought that there are many patients with all the features of heart failure but who have well preserved systolic function-so called diastolic heart failure. Patients with suspected, but unconfirmed, heart failure probably represent the biggest problem and may number 2-3 million in the UK.

Data taken from first time admissions with heart failure in Scotland between 1984 and 1992 show that there has been a steady increase in the number of new admissions with heart failure over the last 15 years and this is true for most, although not all, countries ${ }^{3}$ (table 1 ).

It is easy to explain the increase in admissions for heart failure in the elderly, because we have an aging population. In younger age groups, the increasing incidence of heart failure may be the result of our success in keeping more people alive following myocardial infarctions. Those patients who once would have died are being kept alive, and are subsequently at an increased risk of developing heart failure. Every success in
Table 1 First time discharges with heart failure in Scotland (SHIPS database)

\begin{tabular}{lrrr}
\hline & \multicolumn{1}{c}{1984} & \multicolumn{1}{c}{1988} & \multicolumn{1}{c}{1992} \\
\hline Patients & 9281 & 9852 & 12272 \\
Death & & & \\
$\quad$ Over 3 years & 6231 & 6426 & 7544 \\
$\quad$ Mortality (\%) & 67 & 65 & 61 \\
Readmission over 3 years & & & \\
$\quad$ All cause & 11442 & 14248 & 22182 \\
$\quad$ For CHF & 3232 & 4086 & 6365 \\
CHF bed days & 266075 & 267470 & 320145 \\
\hline
\end{tabular}

$\mathrm{CHF}$, congestive heart failure.

managing myocardial infarction is therefore likely to increase the number of patients with heart failure.

\section{Goals and impact of treatment}

The goals of heart failure treatment are to prolong active life by improving symptoms and delaying progression of the underlying ventricular dysfunction and heart failure. In addition, major morbid events, like infarction and stroke, should be reduced and mortality lowered.

\section{CONSENSUS AND SOLVD}

There are only two landmark placebo controlled studies of angiotensin converting enzyme (ACE) inhibitors in heart failureCONSENSUS and SOLVD. ${ }^{4}{ }^{5}$ In the CONSENSUS study the mean age of patients was 71. All patients had NYHA class IV heart failure and were being treated with diuretics and digoxin. At 12 months there was a 31\% reduction $(p=0.001)$ in mortality in patients treated with an ACE inhibitor compared to placebo. The number of days in hospital was reduced by $23 \%{ }^{4}$

In the SOLVD study patients had an ejection fraction of less than $35 \%$ and were less than 80 years old. ${ }^{5}$ Most patients were being treated with diuretics and digoxin. Compared with CONSENSUS, it was a younger patient population and $57 \%$ of patients had class II heart failure. At 41 months, there was a $16 \%$ reduction $(p=0.0036)$ in total mortality and a $26 \%$ reduction $(p<0.0001)$ in death or worsening heart failure in patients being treated with ACE inhibitors compared to placebo. These results led to ACE inhibitors becoming part of the standard treatment for heart failure.

\section{CURRENT SITUATION}

Although these two studies show the mortality benefits of ACE inhibitors, there is still much room for improvement. For example, the data from the CONSENSUS study demonstrates a mortality rate of $36 \%$ at one year in the treatment group. 
The Scottish discharge database shows that mortality from heart failure has dropped from $67 \%$ in 1984 down to $61 \%$ in 1992 . Although, this is highly significant ( $p=0.000001)$, it is not a great success. ${ }^{3}$

Moreover, patients with heart failure do not die quietly. For the 12000 patients with new-onset heart failure studied in Scotland in 1992, there were many hospital readmissions with a third of a million bed days used for heart failure admissions alone over the following three years. This evidence confirms that heart failure has not been defeated by current treatment. There are still many symptomatic patients and the high level of morbidity and mortality remains a major challenge.

Extending the benefits of ACE inhibitors

There are six strategies that will help extend the benefits of ACE inhibitors in the management of heart failure:

- Initiating treatment and ensuring good patient compliance

- Treating patients sooner

- Adding treatments

- Optimising the dose

- Eliminating confounding treatment

- Examining new disease areas

INITIATION AND COMPLIANCE

In one study in the USA, compliance with digoxin prescribed by a doctor was found to be approximately $10 \% .^{6}$ Ninety per cent of patients were not collecting sufficient prescriptions to be taking the digoxin as instructed by their doctor. Compared to other cardiovascular disease areas, it is remarkable how little information there is on compliance with heart failure treatment.

In the US and the UK, $40-50 \%$ of patients with heart failure who have been through a hospital will be given an ACE inhibitor. ${ }^{78}$ Looking only at those patients in whom there is no clear contraindication for ACE inhibition, $73 \%$ of patients receive ACE inhibitors. ${ }^{9}$

In general practice in the $\mathrm{UK}$, the rate of ACE inhibitor use is approximately $33 \%,{ }^{10}$ which is probably representative of primary care practice worldwide. This poor performance may reflect the sheer size of the public health problem of heart failure.

STARTING TREATMENT SOONER

All the evidence points to the fact that, if there is major systolic dysfunction of the left ventricle, then ACE inhibitor treatment should be considered even before symptoms develop. The HOPE study provides clear evidence that ACE inhibitor treatment should be started early. ${ }^{11}$

ADDING TREATMENTS

Clinical trials of mild to moderate heart failure (the V-HeFT-1 trial, the SOLVD treatment trial, and a meta-analysis of the $\beta$ blocker trials) showed that, compared to digoxin and diuretics alone, the combination of $\beta$ blockers and ACE inhibitors reduces two year mortality from 34\% to $14 \% .^{512} 13$
In a recent meta-analysis combining results from all the ACE inhibitor and $\beta$ blocker trials in heart failure, it was observed that only 21 patients with mild/moderate heart failure needed to be treated for a year to save one life. ${ }^{13}$ There are very few treatments, in any area of medicine, that are this effective.

In severe heart failure it is not so clear whether the clinical trials can be summated. In the RALES trial, if spironolactone was added to an ACE inhibitor, the six month mortality was reduced from $14 \%$ to $10 \% .{ }^{14}$ There was also clear evidence of long term mortality benefit. Thus, at least two treatments can be added to an ACE inhibitor to extend their benefits: $\beta$ blockers and spironolactone.

\section{INTERACTION BETWEEN ACE INHIBITORS AND} $\beta$ BLOCKERS

In the SOLVD trial, if patients were taking a $\beta$ blocker at baseline, there was a $60 \%$ reduction in the risk of hospitalisation for heart failure when an ACE inhibitor was then added. ${ }^{5}$ If the patient was not taking a $\beta$ blocker already, the reduction was only $30 \%$ following ACE inhibitor treatment. So there is evidence of a beneficial interaction between the two drugs.

In the RALES study, if patients were on an ACE inhibitor and $\beta$ blocker at baseline, there was a $58 \%$ reduction in mortality. ${ }^{14}$ If they were only taking an ACE inhibitor but without the $\beta$ blocker present, mortality was reduced by only $27 \%$.

The ELITE-II study suggested that there was really no difference between losartan and captopril in terms of mortality or other important secondary end points, although the angiotensin II antagonist was better tolerated..$^{15}$ However, patients who were on a $\beta$ blocker derived greater benefit from the ACE inhibitor. Again, the ELITE-II study provided some evidence of a $\beta$ blocker/ACE inhibitor synergistic interaction.

\section{OPTIMISING THE DOSE}

The ATLAS study was designed to investigate the optimal dose of ACE inhibitor in patients with heart failure. ${ }^{16}$ It compared the effects of high dose (32.5-25 mg once daily) and low dose (2.5-5 mg once daily) lisinopril on mortality and morbidity in patients with chronic heart failure (NYHA class II-IV). It was a multicentred, randomised, double blind parallel group study in 3164 patients.

There was a trend towards reduced mortality with high dose lisinopril compared to low dose, but it did not achieve significance $(p=0.128)$. Interestingly, in the under 70 s, there was a clear mortality benefit from the use of high dose lisinopril. There also appeared to be a greater benefit in class II patients, but this did not quite achieve significance. There was a highly significant benefit in favour of the high dose in terms of all cause hospitalisation, mainly for cardiovascular reasons and indeed predominantly for heart failure itself (fig 1).

Interestingly, the class II patients, who had very few symptoms, were the ones who really benefited from large doses of ACE inhibitors. An important message from the ATLAS study 
is that patients with mild heart failure need large doses; sick patients should be given the highest dose that can be tolerated.

The ATLAS study suggested that treatment of 1000 patients for three years with high dose lisinopril would save 37 deaths and 395 hospitalisations compared with low dose lisinopril. In the US, this has been calculated to save about $\$ 2$ billion a year. In the UK, which has a much cheaper healthcare system, the strategy would be at least revenue neutral.

\section{ELIMINATING CONFOUNDING TREATMENTS}

Eliminating confounding treatment will become increasingly topical in cardiovascular disease in the next five years, particularly with regard to aspirin. There are growing worries, and growing evidence, that aspirin may be deleterious in patients with heart failure and coronary disease.

One of the first pilot studies to address this question compared warfarin, aspirin, and no antithrombotic treatment in 279 patients, with approximately 600 patient years of follow up. ${ }^{13}$ In the primary outcome of death, myocardial infarction or stoke, there was no real difference, with a $32 \%$ event rate on aspirin versus $26 \%$ in patients receiving no treatment. Looking at the secondary outcomes, there was a significant excess of hospitalisation with aspirin, which is caused by a near doubling in the risk of hospitalisation with worsening heart failure.

There is a growing body of evidence that aspirin can take away most of the benefits of ACE inhibitor treatment. In the SOLVD trial, patients taking aspirin at baseline would have been better off on placebo than enalapril: mortality rates were $31 \%$ with placebo versus $35 \%$ with enalapril.

\section{EXAMINING NEW DISEASE AREAS}

The largest randomised controlled trial evidence we have on diastolic heart failure so far is the V-HeFT-II trial, comparing hydralazine isosorbide with enalapril, and suggesting a mortality benefit with the ACE inhibitor. ${ }^{17}$ There are a number of ongoing studies in this area and in three or four years' time we will have a great deal more information on this syndrome and how it should be treated; at the moment, however, recommendations cannot be made.

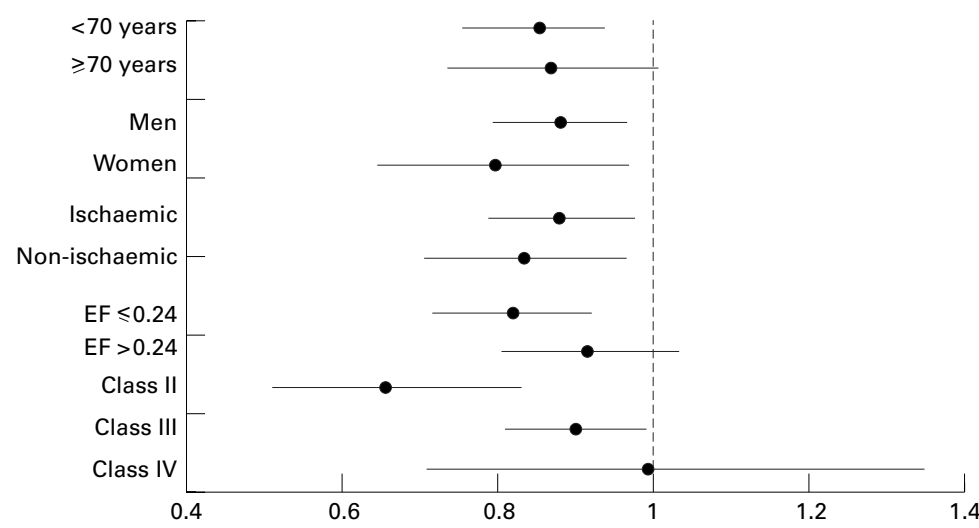

Figure 1 ATLAS study — high dose: low dose hazard ratios for death or hospitalisation for any reason in patient subgroups defined by five pretreatment characteristics.
Trial acronyms

ATLAS: Assessment of Treatment with Lisinopril And Survival

CONSENSUS: Co-operative North

Scandinavian Enalapril Survival study

ELITE II: Evaluation of Losartan in the Elderly-II

HOPE: Heart Outcomes Prevention Evaluation

RALES: Randomised Aldactone Evaluation Study

SOLVD: Studies of Left Ventricular Dysfunction

V-HeFT: Vasodilator Heart Failure Trial

\section{Conclusion}

ACE inhibitors remain one of the cornerstones of the treatment of heart failure. There is clear evidence that higher doses exert greater benefit. They are usually very well tolerated, especially in milder cases. There should be some caution about the use of high doses in the frail elderly patient. There is now fairly conclusive evidence of a synergistic effect with $\beta$ blockers. In sick patients, addition of spironolactone should be considered, even to high dose ACE inhibition. At present, there is no evidence to support the switch to, or indeed the addition of, angiotensin II antagonists.

1 Cowie MR, Mosterd A, Wood DA, et al. The epidemiology of heart failure. Eur Heart f 1999;18:208-25

2 Ho KK, Pinsky JL, Kannel WB et al. The epidemiology of heart failure, the Framingham Study. $\mathcal{F} \mathrm{Am}$ Coll Cardiol 1993;22(4 suppl A):6A-13A.

3 Cleland JG, Gemmed I, Khand A, et al. Prognosis of heart ailure improving? European fournal of Heart Failure 1999;1:229-41.

4 The CONSENSUS Trial Study Group. Effects of enalapril on mortality in severe congestive heart failure: results of the co-operative north Scandinavian enalapril survival study (CONSENSUS). N Engl F Med 1987;316:1429-35.

5 The SOLVD Investigators. Effect of enalapril on survival in patients with reduced left ventricular ejection fractions and

congestive heart failure. N Engl f Med 1991;325:293-302.
Monane M, Bohn RL, Gurwitz JH, et al. Noncompliance with congestive heart failure therapy in the elderly. with congestive

7 Philbin EF, Andreaou C, Rocco TA, et al. Patterns of angiotensin-converting enzyme inhibitor use in congestive heart failure in two community hospitals. Am $\mathcal{F}$ Cardiol 1996;77:832-8

8 Hillis GS, Trent RJ, Winton P, et al. Angiotensin-convertingenzyme inhibitors in the management of cardiac failure: are we ignoring the evidence? Q7M 1996;89:145-50.

9 Hillis GS, Al-Mohammed A, Wood M, et al. Changing patterns of investigation and treatment of cardiac failure in hospital. Heart 1996;76:427-9.

10 Mair FS, Crowley TS, Bundred PE. Prevalence, aetiology and management of heart failure in general practice. $\mathrm{Br} \mathcal{F}$ Gen Pract 1996;46:77-9.

11 Yusuf S, Phil D, Sleight P. Effects of an angiotensinconverting-enzyme inhibitor, ramipril, on cardiovascular events in high-risk patients. $N$ Engl f Med 2000;342:14553.

12 Cohn JN, Archibald DG, Zeische S, et al. Effect of vasodilator therapy on mortalilty in chronic congestive heart failure: results of a Veterans Administrative cooperative study. N Engl f Med 1986;314:1547-52.

13 Cleland JG, McGowan J, Clarke A, et al. The evidence for beta-blockers in heart failure. BMF 1999; 318:824-5.

14 The RALES Investigators. Effectiveness of spironolactone added to an angiotensin-converting enzyme inhibitor and a loop diuretic for severe chronic congestive heart failure. The randomised aldactone evaluation study (RALES). Am 7 Cardiol 1996;78:902-7.

15 Topol E. ACE inhibitors still the drug of choice for heart failure. Lancet 1999; 354:1797.

16 The ATLAS Study Group. Comparative effects of low and high doses of the angiotensin-converting-enzyme inhibitor, lisinopril, on morbidity and mortality in chronic heart failure. Circulation 1999;100:2312-18.

17 Cohn JN, Johnson G, Ziesche S, et al. A comparison of enalapril with hydralazine-isosorbide dinitrate in the treatment of chronic congestive heart failure. N Engl f Med 1991;325: 303-10. 